Conclusion This paper identifies the evidence base for the CSNAT intervention and lessons learnt about delivery of comprehensive, person-centred carer assessment and support for family carers.

\section{P-80 USING PROJECT ECHOTM TO SUPPORT COMPREHENSIVE PERSON-CENTRED CARER ASSESSMENT AND SUPPORT IN HOSPICES}

${ }^{1}$ Janet Diffin, ${ }^{2}$ Gail Ewing, ${ }^{3}$ Gunn Grande. ${ }^{1}$ Hospice UK, London, UK; ${ }^{2}$ Cambridge University, Cambridge, UK; ${ }^{3}$ University of Manchester, Manchester, UK

\subsection{6/bmjspcare-2019-HUKNC.103}

Background Barriers and facilitators to implementation of comprehensive person-centred assessment and support within palliative care have been identified (Ewing \& Grande, 2018). To address the challenges, an online toolkit was developed to provide organisations with structured implementation guidance for the Carer Support Needs Assessment Tool (CSNAT) intervention. However, feedback from practitioners undertaking previous face-to-face CSNAT intervention training and from online toolkit participants highlighted the benefits from follow-up support calls with the opportunity to ask questions and problem solve with their peers, the opportunity to build networks with other organisations completing the training, and having a point of contact to ask questions (Diffin, Ewing, Rowland, Grande, 2018). Currently there is no method for providing this additional support. Project $\mathrm{ECHO}^{\mathrm{TM}}$ (Extension for Community Healthcare Outcomes) is a tele-mentoring programme which uses video-conferencing to deliver best practice guidance and education from specialists, and case-based learning with opportunities for questions and discussion with peers, and therefore could overcome this gap.

Aim To identify if Project $\mathrm{ECHO}^{\mathrm{TM}}$ could be used to establish a community of practice and support implementation of the CSNAT intervention.

Methods and results We will draw on the CSNAT implementation evidence base to illustrate the importance of supporting practitioners to gain knowledge about how to use the CSNAT in practice, and enhance their confidence in carrying out assessments. In addition, we will reflect on the implementation support needed at an organisational level, to include practical guidance and opportunities for peer support. In turn, we will highlight how Project $\mathrm{ECHO}^{\mathrm{TM}}$ could be used to help overcome identified gaps in support through reflection on the core benefits of this methodology to include a curriculum identified by the network participants themselves, case-based learning, and the ability to join remotely.

Conclusion This paper identifies how Project $\mathrm{ECHO}^{\mathrm{TM}}$ could be utilised to establish a community of practice to support implementation and embedding of comprehensive person-centred assessment and support for family carers into everyday practice.

\section{P-81 SUPPORT NEEDS OF INFORMAL CARERS OF PATIENTS WITH COPD AND IMPLICATIONS FOR IMPROVING CARER SUPPORT}

Kerry Micklewright, Morag Farquhar. University of East Anglia, Norwich, UK
Introduction Informal carers play a key supportive role for patients with Chronic Obstructive Pulmonary Disease. The care they provide also plays a vital role in relieving pressure on health and social services. However, caring can have a considerable impact on health and wellbeing and carers may have unidentified support needs that could be a target for intervention. Literature on the support needs of these carers has not been fully synthesized, and our knowledge of the comprehensiveness of the Carer Support Needs Assessment Tool (CSNAT) for these carers is limited.

Methods English language studies published between 19972017 were identified against predetermined inclusion/exclusion criteria through searches of MEDLINE, CINAHL, EMBASE, CDSR, ASSIA, PsycINFO and Scopus. Further studies were identified through searching reference lists and citations of included papers. Papers were critically appraised and data extracted and synthesised by two reviewers. Identified needs were mapped to CSNAT items.

Results 24 studies were included. Results suggest that carers have support needs in a range of domains including physical, social, psychological and spiritual. Many of these needs are unmet. Particular areas of concern relate to: prolonged social isolation, accessing services, emotional support and information needs.

Findings also suggest additional CSNAT items may be required in order to encompass the full range of needs of this group, particularly relating to difficulties within patient-carer relationships and carer-clinician relationships.

Conclusion Based on this review, there is evidence to suggest that COPD carers would benefit from identification and response to their support needs by healthcare professionals but to enable this the CSNAT requires additional items. Future planned work will develop these items with COPD carers.

\section{P-82 WHAT CARERS OF PEOPLE LIVING WITH BREATHLESSNESS WANT TO LEARN ABOUT 'WHAT TO EXPECT IN THE FUTURE'}

${ }^{1}$ Sylvia Barnes, ${ }^{2}$ Gail Ewing, Morag Farquhar ${ }^{1}$. 'University of East Anglia, Norwich, UK; ${ }^{2}$ University of Cambridge, Cambridge, UK

\subsection{6/bmjspcare-2019-HUKNC.105}

Introduction Breathlessness is distressing and disabling, and common in advanced cancer and non-cancer conditions. Informal carers (family members/friends) provide unpaid support, but many lack knowledge and confidence in caring, experiencing anxiety, uncertainty, and helplessness when faced with breathlessness. The Learning about Breathlessness programme $(\mathrm{LaB})$ is addressing this.

LaB1 identified six topics carers want to learn about breathlessness (Farquhar, Penfold, Benson, Lovick et al., 2017). LaB2 is working with carers, patients and healthcare professionals to co-develop and test a prototype web-based educational intervention for carers on breathlessness. Content for five of the six topics was developed, but additional work was needed for the sixth topic, What to expect in the future', to ensure utility and sensitivity.

Methods Two disease-specific focus groups and six interviews were conducted with bereaved carers of people with breathlessness due to cancer or chronic obstructive pulmonary disease (COPD) $(n=12)$. Data were sought on what 